\title{
L'État malien entre négociations et résistances dans la formulation de politiques sur les migrations
}

\section{Sadio Soukouna}

\section{OpenEdition}

\section{Journals}

Édition électronique

URL : https://journals.openedition.org/anthropodev/966

DOI : 10.4000/anthropodev.966

ISSN : 2553-1719

\section{Éditeur}

Presses universitaires de Louvain

Édition imprimée

Date de publication : 1 décembre 2020

Pagination : 69-84

ISBN : 978-2-39061-078-6

ISSN : 2276-2019

Référence électronique

Sadio Soukouna, "L'Etat malien entre négociations et résistances dans la formulation de politiques sur les migrations », Anthropologie \& développement [En ligne], 51 | 2020, mis en ligne le 01 juin 2021 consulté le 01 février 2022. URL : http://journals.openedition.org/anthropodev/966 ; DOI : https:// doi.org/10.4000/anthropodev.966

La revue Anthropologie \& développement est mise à disposition selon les termes de la Licence Creative Commons Attribution 4.0 International. 


\title{
L'État malien entre négociations et résistances dans la formulation de politiques sur les migrations
}

\begin{abstract}
Sadio Soukouna ${ }^{1}$
En analysant la construction de l'action publique au Mali, cet article étudie les mécanismes et les acteurs pluriels engagés dans les négociations et les résistances face aux prescriptions internationales et européennes sur les migrations. Il retrace et analyse la séquence historique du refus malien de signer les accords de réadmission poussés par la France entre 2006 et 2010. Il examine les interactions, rôles, positions et stratégies contrastés des acteurs étatiques et non étatiques, impliqués dans les différentes étapes de la construction de ce problème public jusqu'à son impossible résolution. Il prolonge la réflexion sur la trajectoire singulière d'opposition et d'affirmation de l'État malien par rapport à la France, en montrant ses ambiguïtés et les évolutions de la gouvernance des migrations au Mali, dorénavant influencee par les attentes et pressions de I'Union européenne en matière de sécurisation des frontières.

This paper explores the various processes and stakeholders involved in resistance to international requirements and European migration policies in Mali. It provides analytical insights that help to understand the failure of the French readmission agreement signature between 2006 and 2010 in Mali. It analyzes the contrasting positions and the roles of state and non-state actors involved in the implementation of public policies. It also extends the existing discussions about the unusual path of opposition by the Malian State against the French State by showing the evolution of migration governance in Mali now shaped by the expectations of the European Union in terms of securing European borders.
\end{abstract}

\footnotetext{
${ }^{1}$ Politiste, IRD, UMR développement et sociétés, université Paris 1 Panthéon-Sorbonne, et université des sciences juridiques et politiques de Bamako ; sadio.soukouna@ird.fr
} 


\section{Introduction}

Vous savez, il n'y a pas de véritable partenariat, le gouvernement malien s'en fout du codéveloppement, tout ce qui l'intéresse, c'est les transferts d'argent des migrants. (entretien anonyme avec un ancien représentant de la délégation de l'Union européenne au Mali, mars 2011)

Au-delà de l'amertume dont témoigne cet ancien représentant de la délégation de I'Union européenne (UE) au Mali, cette affirmation datée de 2011 met en avant les difficultés de l'institution à transformer l'État malien en «bon partenaire " dans la gouvernance des migrations. Elle pose la question de l'appropriation et des transferts des programmes de politiques publiques, soumis à des jeux complexes d'imposition, de négociations et d'interactions entre acteurs nationaux et internationaux (Mény, 1993 ; Bergamaschi, 2011 ; Lavigne Delville, 2017). En analysant la construction de l'action publique au Mali, cet article ${ }^{2}$ interroge les difficultés de mise à l'agenda et de négociation des accords de "gestion concertée des flux migratoires » entre la France et le Mali, ainsi que le refus malien de signer ceux-ci dans la période 2006-2010. Il étudie ainsi les mécanismes et les acteurs pluriels engagés dans les négociations et les résistances face aux prescriptions internationales et européennes sur les migrations. Ce refus malien est étudié dans le contexte d'un État pris dans des logiques d'extraversion (Bayart, 1999) et récipiendaire de l'aide internationale, soumis depuis plusieurs années à l'expérimentation des politiques françaises et européennes de contrôle des flux migratoires. Mais il doit aussi être lu à l'aune des mobilisations sur les enjeux migratoires de la part des mouvements diasporiques maliens et d'associations diverses se mobilisant tant à l'intérieur qu'à l'extérieur du pays. II contraste donc avec d'autres choix politiques dans la région comme, par exemple, ceux de l'État sénégalais qui a signé des " accords relatifs à la gestion concertée des flux migratoires » avec la France en 2006 (Fall, 2008 ; Ba et Ndiaye, 2008). Cette différence s'explique principalement par l'importance accordée, dans les représentations collectives, à la migration clandestine au Sénégal. Contrairement au Mali, cette décision de l'État sénégalais met en évidence la convergence d'actions politiques et militantes sénégalaises contre l'émigration clandestine, rejoignant ainsi l'agenda européen de fermeture des frontières (Bouilly, 2008 ; Kabbanji, 2011 ; Dedieu, 2012).

En s'inscrivant dans une approche configurationnelle de l'action publique (Eboko, 2015), cet article analyse la pluralité des acteurs impliqués ainsi que les complexités et conflictualités liées à la mise en œuvre de politiques de contrôle des flux migratoires au Mali. Le cadre spécifique des négociations bilatérales réalisées entre le Mali et la France durant les deux mandats du président Amadou Toumani Touré (2002-2012) offre la possibilité d'analyser l'agencéité, les positionnements et les stratégies variés de différents acteurs qui ont influé sur le rejet des accords de réadmission franco-maliens entre 2006 et 2010. Au regard de la signature par le Mali d'accords similaires avec l'Espagne en 2007, il permet aussi d'interroger les limites de cette résistance malienne. Nous souhaitons ainsi plus

\footnotetext{
${ }^{2}$ Je remercie sincèrement les différents évaluateurs internes et externes dont les remarques ont été très
} utiles à l'amélioration de cet article. 
largement montrer, à travers l'exemple malien, la façon dont des acteurs sociaux cherchent, avec plus ou moins de réussite, sous des contraintes politiques et financières fortes (Pommerolle, 2008 ; Pommerolle et Siméant, 2008), à porter des problèmes publics et à peser sur l'élaboration et la mise en œuvre de politiques dans un contexte où l'État ne détient plus le monopole de ces processus, compte tenu des réseaux, des interdépendances et des jeux d'acteurs nationaux et internationaux (Lascoumes et Le Galès, 2012 ; Lavigne Delville et Saïah, 2015 ; Darbon et Provini, 2018).

Notre enquête adopte une perspective d'analyse sociohistorique en envisageant la mise en œuvre de politiques migratoires au Mali dans une temporalité plus longue. Elle s'appuie sur des entretiens semi-directifs réalisés en France et au Mali de janvier à mars 2011 auprès d'une vingtaine de personnes: représentants diplomatiques de l'UE, des États français et maliens, experts, techniciens en charge de décrypter ces accords, acteurs associatifs, ONG, élus maliens, responsables et chargés de mission de ministères et d'institutions de coopération internationale en charge des migrations au Mali. L'enquête s'appuie aussi sur l'exploitation d'archives, de rapports, d'articles de presse, de documents et notes de services produits sur ces négociations franco-maliennes.

Cet article est structuré en trois parties. Tout d'abord, nous retraçons le contexte historique et la nature des pressions politiques exercées par la France pour impliquer l'État malien dans la mise en œuvre de politiques de contrôle des migrations dès le milieu des années 1990 et la négociation d'accords de réadmission à partir de 2006. Ensuite, nous montrons comment la formulation de politiques migratoires au Mali s'est affranchie du poids des prescriptions des bailleurs internationaux, pour porter une attention au poids des mobilisations et des financements générés par les migrants maliens de France dans la période 2006-2010. Enfin, nous analysons la résistance de l'État malien vis-à-vis de la France en fonction de ses ambiguïtés et des évolutions de la gouvernance des migrations au Mali, dorénavant influencée par les attentes de l'UE en matière de sécurisation des frontières européennes.

\section{Contexte historique ( $\mathrm{xx}^{\mathrm{e}}$-xxI ${ }^{\mathrm{e}}$ siècle) et pressions françaises pour l'expérimentation des politiques de contrôle des flux migratoires}

Au Mali, dès 1996, la mise en œuvre du codéveloppement ${ }^{3}$ est intervenue dans la coopération française dans la région de Kayes ${ }^{4}$. À l'époque, le choix de cette région n'était pas anodin dans la mesure où, sur les 46000 Maliens officiellement recensés en France

\footnotetext{
${ }^{3}$ Le codéveloppement est défini comme toute action de coopération en rapport avec les populations immigrées et le développement des pays d'origine. La politique de codéveloppement repose, dès ses origines, sur le principe d'instrumentalisation de l'aide au développement. Elle ne cessera d'évoluer, durant plusieurs décennies, en fonction des différents objectifs de contrôle de l'immigration et de développement qui lui sont assignés.

${ }^{4}$ Les autorités maliennes ont participé à une première initiative expérimentale nommée le programme de développement local migrations (PDLM). Ce dispositif proposait une aide à la réinsertion en faveur des projets des migrants de retour et des potentiels candidats au retour.
} 
(Daum, 2007a), la majorité venait de la région de Kayes (Lombard, 2008). En 1997, en France, le rapport de bilan et d'orientation sur la politique de codéveloppement liée aux flux migratoires de l'universitaire Sami Naïr a mis en lumière les objectifs du codéveloppement orientés vers le développement des pays d'origine et la maîtrise des flux migratoires (Daum, 2007b; Courtin, 2007; Lacroix, 2009; Naïr, 1997). Au Mali, ces évolutions ont laissé apparaître des injonctions de coopération (Guiraudon, 2010) et l'émergence d'un "forum » constitué d'acteurs, d'idées et de référentiels de politiques publiques sur les migrations (Jobert, 1992 ; Fouilleux, 2000). Nous observons cela en 2000 avec la formalisation du comité franco-malien sur les migrations. Établi sur la base d'une convention bilatérale de codéveloppement, ce comité est devenu le lieu de discussions et de négociations préalables aux initiatives françaises en matière de migration et de développement au Mali. En 2002, à la suite de la signature par le Mali de la convention de financement nommée " mise en œuvre du codéveloppement », ce comité franco-malien a participé à l'élaboration d'un fonds de solidarité prioritaire (FSP) de codéveloppement. Ces financements français s'élevaient à hauteur de 2,6 millions d'euros, pour trois ans, destinés en priorité à la région de Kayes.

Au Mali, les débuts du codéveloppement ont coïncidé avec la création d'institutions ministérielles censées défendre les intérêts des migrants ${ }^{5}$. Un premier lien explicite s'est opéré ainsi entre migration, développement et sécurité au sein du comité franco-malien sur les migrations. Le Conseil de base des Maliens de France (CBMF) est reconnu au Mali comme un démembrement du Haut Conseil des Maliens de I'Extérieur (HCME) ${ }^{6}$. Selon l'ancien président du CBMF, Gaharo Doucouré, la première proposition française a été exprimée en 2002 lors d'une visite au Mali de Nicolas Sarkozy, à l'époque ministre de l'Intérieur (entretien avec Gaharo Doucouré, janvier 2011). Elle portait sur la délégation des expulsions de migrants maliens de France à des policiers maliens. Ce projet aurait fait l'objet d'un refus du gouvernement malien. Malgré cela, le nombre d'expulsions de Maliens depuis la France a continué d'augmenter au début des années 2000 (Daum, 2002).

Au milieu des années 2000, l'État français a développé une stratégie de coopération basée sur la reconnaissance et l'identification des sans-papiers maliens par leur pays d'origine. C'est ainsi que la réadmission a été mise au cœur des échanges entre le Mali et

\footnotetext{
${ }^{5}$ Les premiers jalons de cette reconnaissance étatique ont été posés par le président Alpha Oumar Konaré arrivé au pouvoir en 1992. Avec Amadou Toumani Touré, cet intérêt de l'État pour la migration s'est renforcé avec la création d'un ministère délégué en 2002, puis d'un ministère entier en 2004 qui devient le ministère des Maliens de l'extérieur et de l'Intégration africaine.

${ }^{6}$ Le HCME est un organe consultatif auprès du gouvernement qui fédère les associations et amicales de migrants maliens dans 62 pays. Le CBMF est censé représenter en France toutes les associations maliennes, même si cela n'est pas le cas dans la réalité. L'association a été investie au cours de ces dernières années comme une organisation affinitaire. Les associations s'y sont inscrites en fonction de leur affinité avec les représentants. C'est la raison pour laquelle on retrouve une partie des associations de Maliens répertoriées auprès du CBMF et une autre partie auprès du HCME.
} 
la France, légitimée par la politique de " développement solidaire ${ }^{7}$ " du président Nicolas Sarkozy. Cette politique privilégiait le retour forcé des migrants au moyen d'accords préétablis avec l'État malien. En 2006, au Mali, les négociations ont été conduites autour de deux visions divergentes résumées par Bassirou Diarra, ancien secrétaire général de la présidence au Mali et conseiller du président Amadou Toumani Touré sur les migrations :

Les autorités françaises avaient pour ambition le contrôle des flux migratoires alors que les autorités maliennes y voyaient plutôt des opportunités de financement de projets de développement. (entretien avec Bassirou Diarra, mars 2011)

La politique française de contrôle des flux migratoires s'appuyait donc sur cette logique de dépendance financière du Mali à l'aide française et internationale, puis sur l'instrumentalisation de la relation entre migration et développement orchestrée par la France (Soukouna, 2016 ; Dedieu, 2018). La mise en œuvre de ces accords au Mali reposait principalement sur des contraintes d'appropriation visant à répondre aux logiques du bailleur de fonds. Ces nouvelles modalités de coopération s'inscrivaient dans la ligne de la " coresponsabilité » des États et bailleurs de fonds entérinée, au début des années 2000, par les objectifs du millénaire pour le développement et la déclaration de Paris (Bergamaschi, 2008 ; Lavigne Delville, 2017).

Au Mali, cette coproduction de politiques reposait sur les stratégies d'une pluralité d'acteurs, étatiques et non étatiques, aux rôles variés (techniciens, politiques). Hamidou Bathily, ancien chef de la Cellule technique de codéveloppement au Mali décrit ici sa mission : "Les techniciens avaient pour rôle de se mettre d'accord sur le contenu des accords, les dispositions qu'il fallait mettre dedans " (entretien avec Hamidou Bathily, février 2011). Son rôle consistait donc à réviser les termes des accords mais la prise de décision revenait, selon lui, aux "hommes politiques" qui étaient le ministre de I'Intérieur $^{8}$ pour la France et le ministre des Maliens de l'extérieur ${ }^{9}$ pour le Mali, faisant le lien avec leurs présidents respectifs. Pour piloter ces négociations, des chefs de file ont été désignés sous la supervision des deux ministres. Le président Amadou Toumani Touré a confié ce rôle à Bassirou Diarra, ancien migrant originaire de la région de Kayes, à l'époque conseiller du président malien (Soukouna, 2018). Selon Hamidou Bathily, ces négociations consistaient d'abord à se mettre d'accord sur le nombre de régularisations de Maliens en France, sur la base d'une liste de métiers, en échange d'une collaboration de l'État malien dans le contrôle policier des frontières.

Ces dispositions participaient en réalité à la mise en œuvre de la politique d'immigration « choisie » prônée par la France. Stéphane Darmas, ancien directeur de l'Office français de

\footnotetext{
${ }^{7}$ À cette époque, le président français a mis sur pied un ministère dédié exclusivement au lien entre migration et développement, nommé ministère de l'Immigration, de l'Intégration, de l'Identité nationale et du Développement solidaire. Le "développement solidaire " a été défini par l'État comme une politique qui s'appuie sur l'aide publique au développement et " contribue à une meilleure gestion des flux migratoires ». Source : "L'essentiel sur le Développement solidaire », 2009, rapport du ministère de I'Immigration, de l'Intégration, de l'Identité nationale et du Développement solidaire.

${ }^{8}$ Brice Hortefeux au début des négociations, puis ensuite Éric Besson à partir de 2009

${ }^{9}$ À l'époque Badara Aliou Macalou.
} 
l'immigration et de l'intégration, par exemple, a affirmé avoir été recruté à l'époque pour mettre en œuvre les finalités de cette politique spécifique (entretien avec Stéphane Darmas, mars 2011). Celle-ci reposait sur le mythe que l'immigration économique devrait l'emporter sur les autres formes d'immigration, notamment le regroupement familial, tout en réduisant globalement le solde migratoire des étrangers (De Haas, 2005 ; Héran, 2017).

En contrepartie de cette politique d'immigration « choisie " française, l'État malien était soumis à des obligations d'assouplissement de la délivrance des laissez-passer consulaires autorisant les expulsions depuis la France. Selon Bassirou Diarra, ancien conseiller présidentiel, c'est la surveillance des frontières et surtout le retour des migrants en situation irrégulière dans leur pays d'origine qui étaient à l'origine des réticences de la délégation malienne. Comme l'affirme Seydou Keita, ancien conseiller technique du ministère des Maliens de l'extérieur et de l'Intégration africaine, cette clause représentait une "difficulté pour le président malien d'expliquer à la population qu'il s'associait à la France pour l'expulsion de ses propres compatriotes " (entretien avec Seydou Keita, février 2011). Ces négociations franco-maliennes se caractérisaient donc par leur complexité à plusieurs égards. Elles ont provoqué des contestations qui ont contribué à élargir le débat à la société malienne, et au-delà. Ces oppositions ont mis aussi en évidence l'attention politique portée aux contributions financières des migrants maliens de France à leurs proches restés au pays.

\section{" An t'a signer $!^{10}$ »: le poids des mobilisations et des pressions financières de la diaspora dans la résistance malienne}

Depuis les années 1990, la question de la réadmission est à l'origine de cadres d'interpellation forgés par les migrants maliens qui s'érigent en de redoutables forces contestataires vis-à-vis de leur État jugé défaillant (Lecadet, 2012 ; Gary-Tounkara, 2013 ; Siméant, 2014). Les mobilisations suscitées par la négociation des accords de réadmission entre la France et le Mali permettent de voir comment des arènes publiques s'articulent autour de dynamiques de constitution de problèmes publics (Cefaï, 2009).

\section{La publicisation du problème : entre dénonciation et opposition aux accords de réadmission}

L'Association malienne des expulsés (AME), créée en 1996, a joué un rôle important dans la mise en récit des aspects négatifs des accords de réadmission entre la France et le Mali. Elle a bénéficié du soutien de réseaux nationaux, mais aussi français et européens. Une première opposition aux accords de réadmission a pris forme en 2007, lors d'une visite du ministre français Brice Hortefeux au Mali ${ }^{11}$. En amont, l'AME a diffusé un

\footnotetext{
10 Slogan porté par l'Association malienne des expulsés lors des manifestations contre les accords de réadmission en 2009. II signifie " On ne signe pas ! » en langue bambara.

${ }^{11}$ Sa visite avait pour but d'augmenter la pression sur l'État malien concernant la signature des accords de réadmission. Voir l'article de presse, «Le ministre Français Brice Hortefeux en visite au Mali : Plus de
} 
message d'opposition ${ }^{12}$ à sa visite dans ses réseaux internationaux ${ }^{13}$ et maliens. Elle y a dénoncé les accords tout en exprimant une vive critique à l'encontre des autorités maliennes. Les réactions suscitées par cet appel ont permis, selon Ousmane Diarra, président de l'association, de mobiliser "plus de 300 Maliens dans les rues de Bamako " (entretien avec Ousmane Diarra, mars 2011). Ces mobilisations ${ }^{14}$ de l'AME contre les accords de réadmission se sont maintenues durant toutes les étapes de négociation des accords, poursuivies avec l'arrivée d'une nouvelle délégation française au Mali conduite par le ministre Éric Besson fin mars 2009. En réaction, l'AME s'est alliée au Forum pour un autre Mali, l'association de la militante altermondialiste Aminata Dramane Traoré, afin de redoubler la pression sur les autorités maliennes. Les deux associations ont alors publié une déclaration conjointe demandant aux autorités maliennes de refuser de signer ces accords avec la France. Selon Ousmane Diarra, au même moment, en France, une manifestation a eu lieu à l'appel de la Coordination des sans-papiers de Paris devant le consulat du Mali avec l'objectif de "déjouer ce qui se trame entre les gouvernements français et malien derrière le dos des Maliens sans-papiers de France " (Ousmane Diarra, entretien cité). Ces différentes articulations ont eu des effets transnationaux étudiés par Clara Lecadet (2017), comme la démonstration de l'efficacité des mobilisations conjointes entre pays de séjour et pays d'origine.

Parallèlement à ces manifestations, l'AME est parvenue à mobiliser les élus maliens en réalisant deux auditions à l'Assemblée nationale. Ces discussions furent présentées par Oumar Mariko, député de l'opposition, comme une forme de résistance des élus à ces accords :

Notre positionnement était de ne pas signer car cela provoquerait la pénalisation des petits emplois et c'était l'application de l'immigration "choisie». C'est pour cette raison que j'ai proposé, à l'époque, de rassembler la société civile lors d'une rencontre à l'Assemblée nationale. (entretien avec Oumar Mariko, président du parti politique Solidarité africaine pour la démocratie et l'indépendance (Sadi), mars 2011)

C'est donc une critique non dissimulée et une forte pression sociale interne et externe qui ont perturbé les négociations des accords de réadmission entre la France et le Mali. Parallèlement à ces débats et mobilisations, l'apport financier de la diaspora malienne au pays a constitué une " ressource stratégique » (Lavigne Delville, 2017) que l'État malien souhaitait préserver.

280 Maliens expulsés en 2007 par la France » : https://www.afribone.com/?Le-ministre-Francais-Brice (consulté le 4 juin 2020).

12 Intitulé « L'AME manifeste son opposition à la visite de Brice Hortefeux au Mali ».

${ }^{13}$ Cet appel a été relayé par l'ONG Cimade en France, qui compte parmi ses partenaires.

${ }^{14}$ Durant les négociations, les membres de l'AME ont aussi fait circuler une vingtaine de bus nommés «caravanes citoyennes » qui ont sillonné les différentes communes de la capitale malienne. Selon le président de l'association, cette mobilisation avait pour objectif « d'informer et de sensibiliser les populations sur la politique néfaste de Sarkozy et son plan de signature d'accord avec les pays d'origine des migrants pour faciliter les expulsions ». 


\section{Le poids des financements de la diaspora malienne dans l'échec des négociations franco-maliennes}

Lors de ces négociations, la politique du président malien a porté une attention particulière aux investissements et transferts de fonds générés par les ressortissants maliens de France. II s'agissait de préserver cette source importante de revenus généralement perçue comme orientée vers l'amélioration du bien-être des proches restés au pays et plus fiable que l'aide publique au développement parce que moins volatile et moins cyclique (Gubert, 2000 ; De Haas, 2005). De par leur volume dépassant celui de l'aide au développement, ces fonds représentaient une marge de manœuvre considérable permettant à l'État malien de s'extraire de la logique instrumentale de l'aide au développement durant ces négociations.

Au Mali, l'importance de ces transferts financiers des immigrés a été initialement mise en évidence par l'étude BIAO/BECIS (Gonin, 2001 : 16) de juillet 1982 qui les a estimés à 10 milliards de francs maliens (un peu plus de 15 millions d'euros), à destination de la région de Kayes. En 2007, une étude de la Banque africaine de développement a évalué le poids des transferts de la diaspora sur l'économie nationale à 295 millions d'euros (hors transferts informels), plaçant les migrants au rang de premiers bailleurs de fonds au Mali (Banque africaine de développement, 2007). En 2011, la Banque mondiale a estimé à 473 millions de dollars, soit environ $4 \%$ du produit intérieur brut, le montant global des transferts reçus par le Mali (Bernard et al., 2013 : 229). Leur impact a été récemment réévalué à plus de $10 \%$ du produit intérieur brut, alors que l'aide publique au développement n'en représente que 5 \% (Mouhoud, 2016).

Ensuite, la création d'associations de développement des zones d'origine des migrants maliens de France (Daum, 1998 ; Gonin, 2001 ; Quiminal, 2001 ; Bernard et al., 2014) ainsi que les flux financiers vers leurs zones d'origine ont été envisagés par l'État malien comme permettant une transformation et une amélioration des conditions de vie des locaux. Selon Seydou Keita, ancien conseiller technique du ministère des Maliens de l'extérieur :

La spécificité du Mali, c'est qu'on connaît aussi l'importance de la contribution de nos émigrés par rapport au développement de notre pays. On sait. [...] Nous n'avons rien fait à Kayes, les migrants ont tout construit. (entretien avec Seydou Keita, février 2011)

Ainsi, face au poids économique de la diaspora, la stratégie de l'État malien a consisté à réorienter les négociations vers la question de la régularisation des Maliens de France. Cet argument représentait un moyen de justifier la signature des accords de réadmission sans se mettre en porte-à-faux avec l'opinion publique malienne et les migrants. Ce point fut donc intégré à l'agenda des négociations et fit l'objet de longues discussions à propos du quota de migrants maliens à régulariser annuellement. Selon Bassirou Diarra, ancien conseiller du président malien, le désaccord majeur entre les parties portait sur la proposition malienne de 5000 migrants à régulariser par an, alors que la France ne proposait qu'un quota de 1500 personnes. Les différentes sessions de négociations, réalisées au Mali et en France, de façon alternée, permirent aux deux parties de revoir leurs positions. Ainsi, selon l'ancien conseiller du président malien, le Mali aurait revu à la baisse le nombre de migrants à régulariser « 4000 personnes tandis que la France 
proposait 3200 régularisations annuelles » (entretien avec Bassirou Diarra, mars 2011). II affirme néanmoins qu'aucune suite n'a été donnée à ces discussions suite à l'arrivée d'Éric Besson au ministère de l'Immigration, de l'Intégration, de l'Identité nationale et du Développement solidaire en 2009. La politique menée par ce dernier prônait l'intensification de la lutte contre l'immigration dite illégale. Selon Bassirou Diarra, le ministre français aurait mis un frein aux propositions maliennes en affirmant que la France s'en tenait à sa proposition initiale, soit une régularisation annuelle de 1500 personnes. Ce désaccord a été le principal argument mobilisé par nos différents interlocuteurs pour justifier le blocage et l'arrêt des négociations entre le Mali et la France.

Ces transferts de fonds de la diaspora constituent donc un paramètre non négligeable expliquant les pressions exercées sur l'État malien par les mouvements diasporiques et leurs proches restés au pays. Comme le souligne Bassirou Diarra, les motifs ayant présidé au refus de la signature de ces accords franco-maliens étaient "la prise en compte des intérêts des compatriotes maliens en France » et la crainte "d'avoir toute la diaspora malienne sur le dos » (Bassirou Diarra, entretien cité).

Toutefois, cette résistance de l'État malien face à la signature d'accords de réadmission avec la France se caractérise par son ambiguïté et ses limites. Alors que les tensions sociales étaient à leur comble à propos des accords franco-maliens, le Mali signa des accords de " coopération en matière de régulation et gestion des flux migratoires " avec l'Espagne en 2007 (OCDE/CSAO, 2008). Bien que le Mali figure en 2009 parmi les trois pays d'Afrique subsaharienne les plus représentés en Espagne (Odden, 2010), la signature de ces accords ne suscita pas une importante mobilisation des migrants ni une attention politique et médiatique comme ce fut le cas pour les accords avec la France. De plus, à cette époque, le Mali s'engagea également dans de nouvelles modalités de collaboration avec l'UE qui portaient sur d'éventuels accords de réadmission des ressortissants maliens.

\section{Ambiguiités de la résistance malienne, contre-point espagnol et renouvellement des négociations avec I'Union européenne}

Les migrants maliens d'Espagne, moins bien organisés que ceux de France, n'ont pas impulsé de mobilisation ni d'actions contestataires contre la signature des accords de réadmission avec l'Espagne. Ousmane Diarra, président de l'AME, l'a expliqué par l'idée que " le gouvernement malien aurait précipité la signature de ces accords avant qu'il n'y ait eu de possibilité de contestation de la part des acteurs de la société civile " (entretien avec Ousmane Diarra, mars 2011). Toutefois, selon Daouda Gary-Tounkara (2013), l'impact médiatique et symbolique tant des expulsions d'Espagne que des négociations autour de ces accords serait surtout lié à l'inexistence d'un discours de "dette " coloniale de l'Espagne envers le Mali, contrairement à la France. L'auteur souligne que "l'histoire pèse plus dans la relation Mali/France que dans la relation Mali/Espagne " (GaryTounkara, 2013 : 52). Ces accords, signés le 23 janvier 2007 par le Mali et l'Espagne, se présentaient sous la forme d'une convention bilatérale de coopération en matière d'immigration. Selon Bassirou Diarra, avec ces accords, "l'État espagnol proposait de faciliter la mobilité de 800 travailleurs saisonniers en Espagne avec, en contrepartie, la 
possibilité d'expulser les migrants maliens irréguliers d'Espagne " (Bassirou Diarra, entretien cité). Ces derniers étaient estimés à 22318 personnes en 2009 (Odden, 2010 : 99). Tout comme Gary-Tounkara (2013) le souligne, la contrepartie espagnole de ces accords semblait plus importante, garantissant davantage le maintien d'une migration circulaire d'une main-d'œuvre qualifiée malienne selon les besoins de l'économie espagnole. Toutefois, ces accords entre l'Espagne et le Mali s'inscrivaient dans un contexte de formulation d'une politique migratoire espagnole de plus en plus restrictive, $d^{\prime}$ un pays ayant historiquement accueilli les migrants subsahariens, avec une économie en plein essor et un besoin de main-d'œuvre dans les secteurs formel et informel. Selon Moïse Ballo (2009) cité par Gary-Tounkara (2013), sur la période 2002-2008, l’Espagne a expulsé plus de migrants en situation dite irrégulière vers le Mali que la France (2 572 contre 2 174). Malgré cela, il semblerait que le gouvernement malien ait préféré miser sur la contrepartie offerte par les migrations circulaires facilitées par ces accords, au moment où il ne parvenait pas à s'entendre avec le gouvernement français sur la question des régularisations. Dans la pratique, la mise en œuvre de ces accords espagnols s'est réalisée au détriment du Mali qui n'a pu envoyer au total que 29 travailleurs saisonniers en Espagne en 2009. Peu de temps après, l'Espagne a mis fin à la migration des travailleurs saisonniers maliens pour des raisons de "crise économique ". Pourtant, selon Ousmane Diarra, «les autorités espagnoles ont continué, après cette période, l'expulsion des Maliens sans papiers »(Ousmane Diarra, entretien cité).

Ainsi, la nature et les effets de ces négociations bilatérales avec l'Espagne viennent éclairer les limites de la stratégie malienne de défense des intérêts des migrants. L'absence d'une véritable stratégie de l'État malien sur l'aide internationale, les pressions autour des accords de réadmission et la nécessité de préserver des opportunités de migration donnent beaucoup de marge de manœuvre aux États européens. De plus, le Mali devait faire face à ces négociations avec la France et l'Espagne dans un contexte où I'UE renforçait sa coopération axée sur une approche de plus en plus sécuritaire des migrations.

\section{L'approche européenne et l'implication des pays d'émigration dans la gestion des flux migratoires}

Dès le début des années 1990, la coopération de l'UE en matière d'immigration en provenance des pays tiers a été dominée par un discours sécuritaire (Nyberg-Sørensen, 2012). Cette période coïncidait avec l'émergence d'une "nouvelle " approche du partenariat. Cette dernière promouvait la nécessité d'une "participation des pays d'origine et de transit à la gestion efficace des migrations » (Kunz, $2013: 1227$, traduction libre de l'auteure). Progressivement, les préoccupations de l'UE en matière de contrôle migratoire et de lutte contre l'immigration clandestine sont apparues dans les conclusions de ses différents sommets organisés à partir du début des années 2000. D’abord, dans l'article 13 de l'accord de Cotonou avec les pays d'Afrique, des Caraïbes et du Pacifique (ACP) en 2000 qui oblige l'UE et les pays ACP à reprendre leurs propres citoyens "sans autres formalités ". Ensuite, lors du sommet de Séville en 2002, où l'UE prend un virage 
sécuritaire avec la décision d'utiliser la coopération économique et l'aide au développement pour atteindre ses objectifs de maîtrise des flux migratoires (Lavenex et Kunz, 2008). Les grandes lignes de cette politique sont apparues dans la déclaration conjointe AfriqueUE sur la migration et le développement conclue à Tripoli en 2006. Cette déclaration avance l'idée $d^{\prime}$ '« un partenariat entre les pays d'origine, de transit et de destination pour mieux gérer les migrations de manière globale, holistique et équilibrée, dans un esprit de responsabilité partagée et de coopération ${ }^{15}$ ".

Toutefois, comme le montre Rahel Kunz (2013), ces discours de I'UE sur le partenariat peuvent être compris en fonction de logiques de gouvernementalité. Ils cachent en réalité des technologies néolibérales d'encadrement et de gestion des migrations. L'approche globale sur les migrations de 2006, initiée lors de la conférence euro-africaine de Rabat les 10 et 11 juillet 2006 au Maroc ${ }^{16}$, a concrétisé cette vision en adoptant la conclusion principale que les États signataires devraient « mieux utiliser le potentiel de la migration légale et ses effets bénéfiques sur le développement des pays d'origine et des pays d'accueil " (Lavenex et Kunz, 2008 : 451, traduction libre de l'auteure). En pratique, sa mise en œuvre s'est renforcée autour d'un agenda sécuritaire visant à renforcer le contrôle des frontières européennes.

On observe cette logique au Mali où la mise en œuvre de l'approche européenne s'est concrétisée en 2007 avec la création du Centre d'information et de gestion des migrations (CIGEM). Cet instrument d'encadrement des migrations financé par l'UE visait à mettre en œuvre des activités de sensibilisation et de promotion de la migration légale. La création du CIGEM résulte de la déclaration conjointe sur la migration et le développement signée en 2007 par le Mali, la CEDEAO, la France, l'Espagne et la Commission européenne. Cette dernière a financé le CIGEM à hauteur de « 10 millions d'euros en exprimant le besoin que ce projet pilote soit transposé dans la sous-région en cas de réussite » (Funakawa, 2009 : 6). La mise en œuvre du CIGEM au Mali montre d'abord la forte précipitation dans laquelle a été mise en œuvre ce partenariat. Selon Abdoulaye Konaté, ancien directeur du CIGEM :

Un an après la signature de la convention, le bailleur a tenu à ce que le centre soit ouvert très vite, on a alors satisfait à cette demande, ce qui fait que je me suis retrouvé ici sans savoir comment orienter le projet. On a donc dû improviser... que faire d'autre ? (entretien avec Abdoulaye Konaté, février 2011)

Ce besoin hâtif d'expérimentation du dispositif CIGEM s'est, par ailleurs, accompagné de l'imposition de l'approche de l'UE dans un champ institutionnel malien éclaté. La création du centre a mis en évidence l'intervention d'une multitude d'acteurs dans le paysage malien de gestion des migrations, qui occupent à peu près les mêmes fonctions. On

\footnotetext{
${ }^{15}$ European Union, « Joint Africa-EU Declaration on Migration and Development, Tripoli Declaration », 22 November 2006, 11 p., voir: http://www.unhcr.org/refworld/docid/47fdfb010.html (consulté le 4 juin 2020).

${ }_{16}$ Processus de Rabat, dialogue euro-africain sur la migration et le développement, voir : https:// www.rabat-process.org/fr/a-propos/processus-de-rabat (consulté le 4 juin 2020).
} 
l'observe, par exemple, dans la mise en œuvre des questions de réinsertion des migrants de retour. Entre 2008 et 2010, cette question était gérée à la fois par la délégation générale des Maliens de l'extérieur, le CIGEM et l'ANPE.

À partir de 2010, suite au retrait de la France, l'UE s'est engagée dans le financement du codéveloppement au Mali dans un contexte hostile aux réadmissions. Toutefois, en 2011, la politique de l'UE a pris un tournant particulier avec l'adoption de la stratégie de l'UE pour la sécurité et le développement au Sahel. Cette stratégie élaborée en réaction au contexte d'instabilité au Sahel « vise à trouver la synergie entre les mesures en faveur du développement et celles destinées à améliorer la sécurité de la région ${ }^{17}$ ". Elle sous-tend l'idée que l'amélioration de la sécurité dans la région permettrait de "limiter l'émigration des populations, les risques d'attaques sur le sol européen, les trafics illégaux et les problèmes d'approvisionnement en hydrocarbures "(Pichon, $2016: 3)$. Cette approche envisage la migration comme une menace et montre à quel point « le lien entre migration, développement et sécurité se concentre principalement sur la sécurité de l'Occident aux dépens des autres pays " (Nyberg-Sørensen, $2012:$ 67, traduction libre de l'auteure). À la suite de la crise politique et sécuritaire malienne de 2012, l'UE s'est appuyée sur cette stratégie pour développer un partenariat en termes de gouvernance des migrations avec le nouveau régime d’Ibrahim Boubacar Keïta, arrivé au pouvoir en 2013.

En 2016, les effets de cette nouvelle coopération UE-Mali provoquèrent des remous au sein du gouvernement malien et des protestations dans le milieu associatif malien. Ils intervenaient à la suite d'une déclaration du ministre néerlandais Bert Koenders, président en exercice de I'UE, concernant la signature d'un accord UE-Mali sur l'immigration et la réadmission. Au Mali, les réponses apportées par l'État concernant cette coopération témoignaient d'un jeu d'équilibriste visant à rassurer à la fois les partenaires européens et les migrants maliens (Lecadet, 2017). Toutefois, l'approche de coopération entre I'UE et le Mali maintient l'articulation historique entre migration et développement avec la prise en compte de nouveaux enjeux liés au contexte sécuritaire malien tels que la "paix au Mali ${ }^{18}$ ". De même, cette coopération affiche l'objectif de "renforcer la promotion de la migration légale " déjà amorcée par l'UE avec des financements dédiés à plusieurs régions maliennes ciblées : Kayes, Mopti et les régions du Nord du Mali (Gao, Kidal, Ménaka, Tombouctou, Taoudéni) ${ }^{19}$.

\footnotetext{
${ }^{17}$ Cette stratégie cible en premier le Mali, la Mauritanie et le Niger, avant son élargissement au Burkina Faso et au Tchad en 2014.

18 Journal Le Figaro, 2016, "Un accord Mali-UE pour "gérer" l'immigration », voir: https:// www.lefigaro.fr/flash-actu/2016/04/15/97001-20160415FILWWW00266-un-accord-mali-ue-pour-gerer-Iimmigration.php (consulté le 4 juin 2020).

${ }^{19}$ Revue de journaux maliens, blog du Collectif régional pour la coopération Nord-Sud (CORENS), 2016, " Gérer l'immigration : accord UE-Mali », voir : https://revuedepressecorens.wordpress.com/2016/04/17/ gerer-limmigration-accord-ue-mali/ (consulté le 4 juin 2020).
} 


\section{Conclusion}

Cet article éclaire, d'un point de vue sociohistorique, la mise en œuvre de politiques restrictives sur les migrations au Mali. La nature des décisions politiques prises depuis plusieurs décennies en France et au Mali montrent des trajectoires différenciées avec, d'une part, des mesures se durcissant contre l'immigration et, d'autre part, des politiques de défense des intérêts des migrants maliens de France. Ces divergences, bien que n'ayant pas empêché les deux États de négocier, ont permis de voir les différentes stratégies déployées par les acteurs étatiques et non étatiques maliens pour s'opposer aux politiques de réadmission françaises. L'échec des négociations de ces accords jusqu'en 2010 a aussi permis de montrer la place prépondérante accordée à la rente migratoire par l'État malien. En effet, le refus de l'État était en partie motivé par le fait que les transferts de fonds des migrants maliens apparaissaient comme une "ressource stratégique " privilégiée par rapport à l'aide française au développement. Cette étape historique au Mali a permis d'aller au-delà des lectures d'un État " faible " aux ressources limitées pour montrer les formes de résistances, de contournements et de réinterprétations auxquelles la gouvernance des migrations peut donner lieu sur le continent africain.

Ce refus malien a, par ailleurs, montré comment les États de la région du Sahel continuent d'être la cible d'instruments de gouvernement des mobilités au-delà de leurs frontières. La vision stratégique de I'UE dans cette région africaine prône l'expérimentation de politiques sécuritaires visant à associer la figure des migrants en provenance de ces pays à celle des terroristes. L'instabilité au Sahel a ainsi représenté une fenêtre d'opportunité permettant à l'UE d'imposer son approche sécuritaire auprès des États, même s'il a été déjà démontré que le contrôle des migrations est rarement la réponse aux problèmes de sécurité (Bigo, 2001). Enfin, si le lien entre migration et sécurité n'est pas nouveau, il tend à être plus que jamais présent dans les politiques des États africains et au cœur des stratégies de développement au Sahel poussées par les États membres de l'Union européenne.

\section{Bibliographie}

Ba C.O., Ndiaye A.I., 2008, "L'émigration clandestine sénégalaise ", Asylon(s), n³, http:// www.reseau-terra.eu/article717.html (consulté le 4 juin 2020).

Ballo M., 2009, « Migration au Mali : profil 2009 », Genève, OIM, 129 p.

Banque africaine de développement, 2007, «Les transferts des fonds des migrants, un enjeu de développement », rapport BAD, $85 \mathrm{p}$.

Bayart J.-F., 1999, "L'Afrique dans le monde : une histoire d'extraversion », Critique internationale, $\mathrm{n}^{\circ}$ 5, pp. 97-120.

Bergamaschi I., 2008, " Mali : Patterns and Limits of Donor-driven Ownership », in L. Withfield (ed.), The politics of Aid: African strategies for dealing with donors, London, Oxford University Press, pp. 217-242.

Bergamaschi I., 2011, " "Appropriation" et "lutte contre la pauvreté" au Mali », Revue Tiers Monde, $n^{\circ} 205$, pp. 135-150. 
Bernard C., Chauvet L., Gubert F., Mercier M., Mesplé-Somps S., 2013, " La dynamique associative des Maliens de l'extérieur: enseignements tirés de deux dispositifs d'enquête originaux ", working paper, Paris, Dial, $11 \mathrm{p}$.

Bernard C., Chauvet L., Gubert F., Mercier M., Mesplé-Somps S., 2014, « La dynamique associative des Maliens de l'extérieur: enseignements tirés de deux dispositifs d'enquête originaux ", Revue européenne des migrations internationales, $\mathrm{n}^{\circ}$ 3-4, pp. 109-137.

Bigo D., 2001, "La voie militaire de la "guerre au terrorisme" et ses enjeux ", Cultures \& Conflits, $n^{\circ} 44$, pp. 5-18.

Bouilly E., 2008, "Les enjeux féminins de la migration masculine ", Politique africaine, $\mathrm{n}^{\circ} 1$, pp. 16 31.

Cefaï D., 2009, « Comment se mobilise-t-on ? L'apport d'une approche pragmatiste à la sociologie de I'action collective ", Sociologie et Sociétés, n² 2, pp. 245-269.

Courtin C., 2007, "Le codéveloppement: un alibi pour des politiques migratoires restrictives», Revue internationale et stratégique, $\mathrm{n}^{\circ} 68, \mathrm{pp} .43-47$.

Darbon D., Provini O., 2018, " "Penser I'action publique" en contextes africains », Gouvernement et action publique, $n^{\circ} 2$, pp. 9-29.

Daum C., 1998, Les associations de Maliens en France, Paris, Karthala.

Daum C., 2002, "Aides au "retour volontaire" et réinsertion au Mali : un bilan critique », Hommes \& Migrations, $\mathrm{n}^{\circ} 1$, pp. 40-48.

Daum C., 2007a, "Migration, retour, non-retour et changement social dans le pays d'origine ", in V. Petit (éd.), Migrations internationales de retour et pays d'origine, Paris, Ceped, pp. 157168.

Daum C., 2007b, «Le codéveloppement, grandeur et décadence d'une aspiration généreuse », Revue internationale et stratégique, $\mathrm{n}^{\circ} 4$, pp. 49-59.

Dedieu J.-P., 2012, La parole immigrée, Paris, Klincksieck.

Dedieu J.-P., 2018, "The Rise of the Migration-Development Nexus in Francophone Sub-Saharan Africa, 1960-2010 ", African Studies Review, n 1, pp. 83-108.

De Haas H., 2005, "International migration, remittances and development: myths and facts ", Third World Quarterly, n 8, pp. 1269-1284.

Eboko F., 2015, Repenser l'action publique en Afrique. Du sida à l'analyse de la globalisation des politiques publiques, Paris, Karthala.

Fall P.D., 2008, " Migration internationale et développement local dans le Nguènar sénégalais », in M.C. Diop (éd.), Le Sénégal des migrations. Mobilités, identités et sociétés, Paris, Karthala, pp. $195-210$.

Fouilleux È., 2000, « Entre production et institutionnalisation des idées. La réforme de la Politique agricole commune ", Revue française de science politique, $\mathrm{n}^{\circ}$ 2, pp. 277-306.

Funakawa N., 2009, «Le CIGEM, Centre d'information et de gestion des migrations (Mali) : sa place face aux défis des politiques migratoires ", mémoire de master 2 Migrations internationales, université de Poitiers,162 p. 
Gary-Tounkara D., 2013, "La gestion des migrations de retour, un paramètre négligé de la grille $\mathrm{d}^{\prime}$ analyse de la crise malienne ", Politique africaine, $n^{\circ} 2$, pp. 47-68.

Gonin P., 2001, "Les migrations venant du bassin du fleuve Sénégal vers I'Union européenne ", Facteurs d'émigration, politiques d'immigration, colloque du 23 mars 2001, Bruxelles, Centre pour l'égalité des chances et la lutte contre le racisme, pp. 57-86.

Gubert F., 2000, "Migration as a collective risk-coping strategy. Evidence from the Kayes area (Western Mali) ", thèse de doctorat, université d'Auvergne, $288 \mathrm{p}$.

Guiraudon V., 2010, "Les effets de l'européanisation des politiques d'immigration et d'asile », Politique européenne, $\mathrm{n}^{\circ}$ 2, pp. 7-32.

Héran F., 2017, « Avec l'immigration. Mesurer, débattre, agir », Paris, La Découverte.

Jobert B., 1992, «Représentations sociales, controverses et débats dans la conduite des politiques publiques ", Revue française de science politique, $\mathrm{n}^{\circ} 2$, pp. 219-234.

Kabbanji L., 2011, Politiques migratoires en Afrique de l'Ouest, Paris, Karthala.

Kunz R., 2013, "Governing International Migration through Partnership ", Third World Quarterly, $\mathrm{n}^{\circ} 7$, pp. 1227-1246.

Lacroix T., 2009, «Migration, Développement, Codéveloppement: quels acteurs pour quels discours ? ", rapport de synthèse européen, Informer sur les migrations et le développement (IDEM), http://halshs.archives-ouvertes.fr/halshs-00820369 (consulté le 4 juin 2020).

Lascoumes P., Le Galès P., 2012, Sociologie de l'action publique, Paris, Armand Colin.

Lavenex S., Kunz R., 2008, "The Migration-Development Nexus in EU External Relations », Journal of European Integration, $\mathrm{n}^{\circ} 3$, pp. 439-457.

Lavigne Delville P., 2017, "Regards sur l'action publique en Afrique. Introduction », Anthropologie \& développement, $n^{\circ} 45, \mathrm{pp} .13-22$.

Lavigne Delville P., Saïah C., 2015, "Fonder sa légitimité par le plaidoyer ? ", Revue Tiers Monde, $\mathrm{n}^{\circ} 4$, pp. 103-122.

Lecadet C., 2012, «Expulsions et prises de parole au Mali : quand le politique se récrie en ses marges ", Le sujet dans la cité, $\mathrm{n}^{\circ} 1$, pp. 113-127.

Lecadet C., 2017, « Accords de réadmission : tensions et ripostes », Plein droit, n³, pp. 15-18.

Lombard J., 2008, « Kayes, ville ouverte », Autrepart, n³, pp. 91-107.

Mény Y., 1993, Les politiques de mimétisme institutionnel, Paris, L'Harmattan.

Mouhoud E.M., 2016, «Les transferts d'épargne des migrants vers les pays d'origine: un enjeu économique pour le développement ", Revue d'économie financière, $\mathrm{n}^{\circ} 2$, pp. 223-238.

Naïr S., 1997, " Bilan et orientation sur la politique de codéveloppement liée aux flux migratoires », rapport mission interministérielle migrations/codéveloppement, Paris, La Documentation française, $59 \mathrm{p}$.

Nyberg-Sørensen N., 2012, "Revisiting the Migration-Development Nexus: From Social Networks and Remittances to Markets for Migration Control: Revisiting the migration-development nexus ", International Migration, $\mathrm{n}^{\circ} 3$, pp. 61-76. 
OCDE/CSAO, 2008, "Mobilités ouest-africaines et politiques migratoires des pays de l'OCDE ", Cahiers de l'Afrique de l'Ouest, Éditions OCDE, Paris, $149 \mathrm{p}$.

Odden G., 2010, "Parcours et projets des migrants subsahariens en Espagne », Hommes \& Migrations, $\mathrm{n}^{\circ} 1286-1287$, pp. 98-107.

Pichon E., 2016, «Briefing, Sahel Stratégie de l'UE pour la sécurité et le développement », Service de recherche du Parlement européen, $8 \mathrm{p}$.

Pommerolle M.-E., 2008, "La démobilisation collective au Cameroun : entre régime postautoritaire et militantisme extraverti », Critique internationale, $n^{\circ} 3$, pp. 73-94.

Pommerolle M.-E., Siméant J., 2008, « Voix africaines au Forum social mondial de Nairobi. Les chemins transnationaux des militantismes africains $»$, Cultures \& Conflits, $n^{\circ} 70$, pp. 129-149.

Quiminal C., 2001, "Tradition, migration et innovation : la construction d'un marché dans la région de Kayes (Mali) ", Journal des anthropologues. Association française des anthropologues, ${ }^{\circ} 84$, pp. 61-76.

Siméant J., 2014, Contester au Mali, Paris, Karthala.

Soukouna S., 2016, "Les bâtisseurs locaux du lien entre migration et développement: la coopération décentralisée d'île-de-France au prisme des alliances stratégiques entre migrants maliens et pouvoirs locaux dans la région de Kayes au Mali ", thèse de doctorat en science politique, université Paris 1 Panthéon-Sorbonne, $566 \mathrm{p}$.

Soukouna S., 2018, "L'État malien sous l'influence du militantisme en faveur de la cause des migrants ", in L. Chauvet, F. Gubert, T. Jaulin, S. Mesplé-Somps (éd.), Les migrants acteurs des changements politiques en Afrique?, Belgique, De Boeck Supérieur, pp. 177-191. 